Radiation and thermal power of all fission products with long half-lives (more than 60 ooo years) are many orders of magnitude smaller than those of all other fission products in waste that has been stored for several years. Furthermore, long-lived fission products have almost no radiation other than $\beta$-radiation. Future research is necessary as to whether and to what extent such long-lived isotopes, and possibly other isotopes (e.g. ${ }^{239} \mathrm{Pu}$ or ${ }^{14} \mathrm{C}$ ), have to be separated and as to how it could be done in the safest and most economical way. The technology of separating and recycling ${ }^{239} \mathrm{Pu}$, an extremely valuable fissionable fuel, is being developed in view of the increasing importance of breeder reactors. The separate disposal of long-lived isotopes would not raise serious thermal or handling problems; for example, they could be deposited in a highly concentrated form into a deep geological formation.

Should the waste be retrievable or not? That is ultimately a philosophical question. Which is more reliable, man or Nature? Should we trust that our descendants will have sufficient knowledge and goodwill to keep the waste safe and not misuse it - or should we rely more on Nature not to bring the waste into the biosphere by unexpected catastrophic events?

The proposed ice-sheet disposal-be it in deep ice layers or near the surface-avoids the main dangers of both aspects. Under normal glaciological conditions the waste containers are practically irretrievable from the beginning (deep-layer deposit) or after some centuries (near-surface deposit). If, however, a catastrophic climatic change should melt away the ice sheets very quickly, the ablation melts off one after the other of the upper layers while the deep layers still remain cold. Under these circumstances the containers are "self-retrieving": they come to the surface of the ice or of the ice-freed bedrock and can easily be picked up. Further research on such a melt-out process and on the durability of the waste containers and their solidified contents should be carried out.

\title{
CREEP INSTABILITY OF ICE SHEETS
}

\author{
Garry K. C. Clarke
}

(Department of Geophysics and Astronomy, University of British Columbia, Vancouver, British Columbia V6T i W5, Canada)

Abstract. The equation governing the growth or decay of a temperature perturbation $T^{\prime}$ in an ice slab under shear stress $\sigma_{x y}$ is

$$
\frac{\partial^{2} T^{\prime}}{\partial y^{2}}-\frac{V \partial T^{\prime}}{\kappa \partial y}+\frac{a T^{\prime}}{\kappa}=\frac{\mathrm{I}}{\kappa} \frac{\partial T^{\prime}}{\partial t}
$$

where $K$ and $\kappa$ are respectively the thermal conductivity and diffusivity of ice, $V$ is the advection velocity normal to the bed and

$$
a=\frac{\partial}{\partial T}\left[{ }_{2} B_{0} \exp (-Q / R T) \sigma_{x y}{ }^{n+1}\right]
$$

is the rate of increase of strain heating with temperature assuming a power law for flow. For a slab of infinite thickness under constant stress and at constant ambient temperature $T$, Fourier analysis gives $-k^{2}+a \mid k<0$ as the condition for stability where $k$ is the wave number of a sinusoidal perturbation. When the slab has finite thickness the stability depends on the sign of the eigenvalues $\lambda_{m}$ of the perturbation equation and on the boundary condition at the ice-rock interface. In general the eigenfunctions and eigenvalues must be found by approximate methods such as the Rayleigh-Ritz procedure but in the case where the stress and ambient temperature are constant over the slab thickness and there is no advection the eigenfunctions are either sines or cosines depending on the boundary conditions. 
In this special case the stability condition is

if the bed is frozen and

$$
\lambda_{m}=-\left[\frac{(2 m+\mathrm{I})}{2 h} \pi\right]^{2}+\frac{a}{K}<0 \quad m=0, \mathrm{I}, 2, \ldots
$$

$$
\lambda_{m}=-\left(\frac{m \pi}{h}\right)^{2}+\frac{a}{K}<0 \quad m=\mathrm{I}, 2,3, \ldots
$$

if it is at the melting point. The eigenvalue associated with the smallest value of $m$ is the least stable so the maximum stable thickness is thus $h=\frac{1}{2} \pi(a / K)^{\frac{1}{2}}$ if the bed is frozen or $h=\pi(a / K)^{\frac{1}{2}}$ if it is at the melting point. For typical flow-law parameters these depths are around $250 \mathrm{~m}$ and $500 \mathrm{~m}$ respectively. The eigenvalues are related in a simple way to the growth or decay rates of the eigenfunctions: $\left(\kappa \lambda_{m}\right)^{-1}$ is the time constant for the $m$ th eigenfunction.

Depth-dependent stress, temperature, and advection have a marked effect on stability. A slab in which stress and temperature increase to values $\sigma_{\mathrm{B}}$ and $T_{\mathrm{B}}$ at the bed is considerably more stable than a slab held at constant stress $\sigma_{\mathrm{B}}$ and a constant temperature $\mathcal{T}_{\mathrm{B}}$. Advection normal to the bed also has a major influence on stability. If the advection velocity is taken to vary linearly with depth and the bed is frozen, the effect of upward advection is to decrease stability and of downward advection to increase it. When the bed is temperate the effect of advection is more complex: downward advection increases stability but upward advection may increase or decrease it depending on the magnitude of the advection velocity.

\section{DISCUSSION}

W. F. Budd: Have you examined real situation values in your analysis to see the limits for which you can obtain stable solutions?

G. K. C. Clanke: I have just begun to do this and would like to satisfy myself that the numerical methods used to compute eigenvalues are sound and to test a range of flow laws before making generalizations about the region of stability.

\section{ICE SLIDING AND FRICTION EXPERIMENTS}

W. F. BudD

(Antarctic Division, Department of Science, 568 St Kilda Road, Melbourne, Victoria 3oo4, Australia)

Abstract. We are interested in studying the processes of sliding of ice over a variety of rock surfaces with the object of determining an empirical relation for the basal shear stress appropriate for glaciers. The variables to be considered include: normal stress $\mathcal{N}$, shear stress $\tau$, surface roughness $r$, sliding velocity $V$, temperature $\theta$, water at the interface, and the presence of debris. The roughness is considered to be a function of two variables; the scale or wavelength $\lambda$, and the shape or slope of the roughness $a / \lambda$, where $a$ is the amplitude of the variations of that scale.

The experiments are to be carried out in three main phases viz:

(i) Limiting friction tests for no acceleration. For these the ice is placed on a slab and loaded with a normal stress, then the shear stress increased until acceleration occurs. So far the results suggest that for a given roughness the limiting shear stress is linearly related to the normal stress. Typical magnitudes for the limiting coefficient of friction are $\mu \approx 0.35$ for rough slabs. 